\title{
Nanostructured Architectures Promote the Mesenchymal-Epithelial Transition for Invasive Cells
}

\author{
Zongjie Wang ${ }^{1,2}$, Fan Xia ${ }^{3}$, Mahmoud Labib ${ }^{3}$, Moloud Ahmadi ${ }^{3}$, Haijie Chen ${ }^{1}$, \\ Jagotamoy Das ${ }^{3}$, Sharif U. Ahmed ${ }^{3}$, Stéphane Angers ${ }^{3}$, Edward H. Sargent ${ }^{1}$ and \\ Shana O. Kelley $y^{2,3,4^{*}}$ \\ ${ }^{1}$ The Edward S. Rogers Sr. Department of Electrical \& Computer Engineering, University of \\ Toronto, Toronto, M5S 3G4, Canada. ${ }^{2}$ Institute for Biomaterials and Biomedical Engineering, \\ University of Toronto, Toronto, M5S 3G9, Canada. ${ }^{3}$ Department of Pharmaceutical Sciences, \\ Leslie Dan Faculty of Pharmacy, University of Toronto, Toronto, M5S 3M2, Canada. ${ }^{4}$ Department \\ of Biochemistry, Faculty of Medicine, University of Toronto, Toronto, M5S 1A8, Canada. \\ "Correspondence to: shana.kelley@utoronto.ca
}

Tables

Supporting Data
Page S2

Page S6 


\section{Tables}

Table S1. Antibodies used for flow cytometry

\begin{tabular}{|l|l|l|l|l|l|l|}
\hline Antibody & Host & Conjugation & Dilution & Duration & Supplier & Cat. \# \\
\hline Vimentin & Rabbit & AF 488 & $1: 100$ & 1 hour & Abcam & ab185030 \\
\hline Cytokeratin & Mouse & AF 488 & $1: 100$ & 1 hour & Abcam & ab187773 \\
\hline E-Cadherin & Mouse & AF 488 & $1: 20$ & 1 hour & BD & 563570 \\
\hline N-Cadherin & Rabbit & AF 647 & $1: 100$ & 1 hour & Abcam & ab195186 \\
\hline Ki67 & Mouse & AF 647 & $1: 20$ & 1 hour & BD & 561126 \\
\hline FOXA2 & Mouse & PE & $1: 20$ & 1 hour & BD & 561589 \\
\hline C-kit & Mouse & PerCP-Cy5.5 & $1: 20$ & 1 hour & BD & 562094 \\
\hline RhoA & Mouse & Unconjugated & $1: 100$ & 1 hour & Abcam & ab54835 \\
\hline Cdc42 & Rabbit & Unconjugated & $1: 100$ & 1 hour & Abcam & ab187643 \\
\hline Rac1 & Mouse & Unconjugated & $1: 100$ & 1 hour & Abcam & ab33186 \\
\hline Anti-Rabbit & Donkey & AF 488 & $1: 200$ & 30 min & Abcam & ab150073 \\
\hline Anti-Mouse & Donkey & AF 647 & $1: 200$ & 30 min & Abcam & ab150107 \\
\hline Oct4 & Mouse & PE & $1: 11$ & 1 hour & Miltenyi & $130-105-554$ \\
\hline Nanog & Mouse & APC & $1: 50$ & 1 hour & Miltenyi & $130-120-704$ \\
\hline CD44 & Mouse & Biotin & $1: 20$ & 30 min & BD & 555477 \\
\hline Streptavidin & N/A & AF 488 & $1: 100$ & 30 min & Invitrogen & S32354 \\
\hline NCAM & Mouse & PE & $1: 50$ & 30 min & Miltenyi & $130-113-312$ \\
\hline THY1 & Mouse & APC & $1: 50$ & 30 min & Miltenyi & $130-114-903$ \\
\hline Snail & Rabbit & Unconjugated & $1: 50$ & 1 hour & Abcam & ab82846 \\
\hline Integrin $\beta 1$ & Mouse & Unconjugated & $1: 50$ & 1 hour & Abcam & ab24693 \\
\hline pFAK & Rabbit & Unconjugated & $1: 50$ & 1 hour & Invitrogen & 700255 \\
\hline ICAM1 & Mouse & Unconjugated & $1: 50$ & 1 hour & Abcam & ab2213 \\
\hline Anti-Mouse & Donkey & AF 488 & $1: 200$ & 30 min & Abcam & ab150105 \\
\hline Anti-Rabbit & Donkey & AF 647 & $1: 200$ & 30 min & Abcam & ab150075 \\
\hline
\end{tabular}

AF: Alexa Fluor, PE: phycoerythrin, PerCP: peridinin chlorophyll protein complex, Cy: cyanine, APC: allophycocyanin.

Table S2. Antibodies used for immunofluorescence

\begin{tabular}{|l|l|l|l|l|l|l|}
\hline Antibody & Host & Conjugation & Dilution & Duration & Supplier & Cat. \# \\
\hline Vimentin & Rabbit & AF 488 & $1: 100$ & 1 hour & Abcam & ab185030 \\
\hline Cytokeratin & Mouse & APC & $1: 50$ & 1 hour & GeneTex & GTX80205 \\
\hline E-Cadherin & Mouse & AF 488 & $1: 20$ & 1 hour & BD & 563570 \\
\hline
\end{tabular}




\begin{tabular}{|l|l|l|l|l|l|l|}
\hline N-Cadherin & Mouse & AF 647 & $1: 20$ & 1 hour & BD & 563434 \\
\hline Anti-Rabbit & Donkey & AF 488 & $1: 200$ & 30 min & Abcam & ab150073 \\
\hline Anti-Mouse & Donkey & AF 647 & $1: 200$ & 30 min & Abcam & ab150107 \\
\hline DAPI & N/A & N/A & 4 drops/mL & 30 min & Invitrogen & R37606 \\
\hline F-actin & N/A & AF 555 & $7: 500$ & 30 min & Cytoskeleton & PHDH1 \\
\hline Integrin $\beta 1$ & Mouse & Unconjugated & $1: 50$ & 2 hours & Abcam & ab24693 \\
\hline Anti-Mouse & Donkey & AF 488 & $1: 200$ & 1 hour & Abcam & ab150105 \\
\hline
\end{tabular}

AF: Alexa Fluor, APC: allophycocyanin

Table S3. Antibodies used for western blotting

\begin{tabular}{|l|l|l|l|l|l|l|}
\hline Antibody & Host & Conjugation & Dilution & Duration & Supplier & Cat. \# \\
\hline Cytokeratin & Mouse & Unconjugated & $1: 1000$ & 2 hours & CST & $4545 S$ \\
\hline GAPDH & Rabbit & Unconjugated & $1: 1000$ & 2 hours & CST & $2118 S$ \\
\hline Vimentin & Rabbit & HRP & $1: 1000$ & 2 hours & CST & $12826 S$ \\
\hline E-Cadherin & Rabbit & Unconjugated & $1: 1000$ & 2 hours & CST & $3195 S$ \\
\hline N-Cadherin & Rabbit & Unconjugated & $1: 1000$ & 2 hours & CST & $12115 S$ \\
\hline $\begin{array}{l}\text { Phospho GSK- } \\
3\end{array}$ & Rabbit & Unconjugated & $1: 1000$ & 2 hours & CST & $8566 S$ \\
\hline GSK-3 & Rabbit & Unconjugated & $1: 1000$ & 2 hours & CST & $4337 S$ \\
\hline GAPDH & Mouse & HRP & $1: 1000$ & 2 hours & CST & $51332 S$ \\
\hline Anti-Rabbit & Goat & HRP & $1: 1000$ & 1 hour & CST & 7074S \\
\hline Anti-Mouse & Horse & HRP & $1: 1000$ & 1 hour & CST & 7076S \\
\hline
\end{tabular}

HRP: horseradish peroxidase

Table S4. Primers used for qPCR

\begin{tabular}{|l|l|l|}
\hline Gene & Forward primer & Reverse primer \\
\hline E-Cadherin & ATTTTCCCTCGACACCCGAT & TCCCAGGCGTAGACCAAGA \\
\hline $\begin{array}{l}\text { N-Cadherin } \\
18\end{array}$ & TTTGATGGAGGTCTCCTAACACC & ACGTTTAACACGTTGGAAATGTG \\
\hline Vimentin & CCTGTGAAGTGGATGCCCTTA & CAACGGCAAAGTTCTCTTCCA \\
\hline $\begin{array}{l}\text { ZO-1 } \\
(\text { TJAP-1 })\end{array}$ & TGCAACAAGTCCCACTTCCG & ATGTTTCCGAACCATGTCCTG \\
\hline ICAM-1 & GACTAAGCCAAGAGGAAGGAGCAA & TCAGCATACCCAATAGGCAGCAAG \\
\hline ZEB1 & GAAAATGAGCAAAACCATGATCCTA & CAGGT GCCTCAGGAAAAATGA \\
\hline ZEB2 & TTCCATTGCTGTGGGCCTT & TTGTGGGAGGGTTACTGTTGG \\
\hline GAPDH & CATGAGAAGTATGACAACAGCCT & AGTCCTTCCACGATACCAAAGT \\
\hline
\end{tabular}


Table S5. Phosphoro-kinase array configuration

\begin{tabular}{|c|c|c|}
\hline Spot & Marker & Phosphorylation Site \\
\hline $\mathrm{A} 1, \mathrm{~A} 2$ & Reference & $N / A$ \\
\hline $\mathrm{A} 3, \mathrm{~A} 4$ & p38a & T180/Y182 \\
\hline $\mathrm{A} 5, \mathrm{~A} 6$ & ERK $1 / 2$ & T202/Y204, T185/Y187 \\
\hline A7, A8 & JNK 1/2/3 & T183/Y185. T221/Y223 \\
\hline A9, A10 & GSK-3 $\alpha / \beta$ & S21/S9 \\
\hline A13, A14 & p53 & S392 \\
\hline A17, A18 & Reference & $\mathrm{N} / \mathrm{A}$ \\
\hline B3, B4 & EGFR & Y1086 \\
\hline $\mathrm{B} 5, \mathrm{~B} 6$ & MSK1/2 & S376/S360 \\
\hline B7, B8 & AMPKa1 & T183 \\
\hline B9, B10 & Akt $1 / 2 / 3$ & S473 \\
\hline B11, B12 & Akt $1 / 2 / 3$ & T308 \\
\hline B13, B14 & p53 & S46 \\
\hline $\mathrm{C} 1, \mathrm{C} 2$ & TOR & S2448 \\
\hline $\mathrm{C} 3, \mathrm{C} 4$ & CREB & S133 \\
\hline $\mathrm{C} 5, \mathrm{C} 6$ & HSP27 & S78/S82 \\
\hline $\mathrm{C} 7, \mathrm{C} 8$ & AMPKa2 & T172 \\
\hline C9, C10 & $\beta$-Catenin & N/A \\
\hline C11, C12 & p70 S6 Kinase & T389 \\
\hline C13, C14 & p53 & S15 \\
\hline C15, C16 & c-Jun & S63 \\
\hline $\mathrm{D} 1, \mathrm{D} 2$ & Src & Y419 \\
\hline $\mathrm{D} 3, \mathrm{D} 4$ & Lyn & Y397 \\
\hline D5, D6 & Lck & Y394 \\
\hline D7, D8 & STAT2 & Y689 \\
\hline D9, D10 & STAT5a & Y694 \\
\hline D11, D12 & p70 S6 Kinase & T421/S424 \\
\hline D13, D14 & RSK $1 / 2 / 3$ & S380/S386/S377 \\
\hline D15, D16 & eNOS & S1177 \\
\hline E1, E2 & Fyn & Y420 \\
\hline $\mathrm{E} 3, \mathrm{E} 4$ & Yes & Y426 \\
\hline E5, E6 & Fgr & Y412 \\
\hline E7, E8 & STAT6 & Y641 \\
\hline
\end{tabular}




\begin{tabular}{|l|l|l|}
\hline E9, E10 & STAT5b & Y699 \\
\hline E11, E12 & STAT3 & Y705 \\
\hline E13, E14 & p27 & T198 \\
\hline E15, E16 & PLC-Y1 & Y783 \\
\hline F1, F2 & Hck & Y411 \\
\hline F3, F4 & Chk-2 & T68 \\
\hline F5, F6 & FAK & Y397 \\
\hline F7, F8 & PDGFR & Y751 \\
\hline F9, F10 & STAT5a/b & Y694/Y699 \\
\hline F11, F12 & STAT3 & S727 \\
\hline F13, F14 & WNK1 & T60 \\
\hline F15, F16 & PYK2 & Y402 \\
\hline G1, G2 & Reference & N/A \\
\hline G3, G4 & PRAS40 & T246 \\
\hline G9, G10 & Negative Control & N/A \\
\hline G11, G12 & HSP60 & N/A \\
\hline G17, G18 & Negative Control & N/A \\
\hline
\end{tabular}




\section{Supporting Figures}

\section{Bare gold}

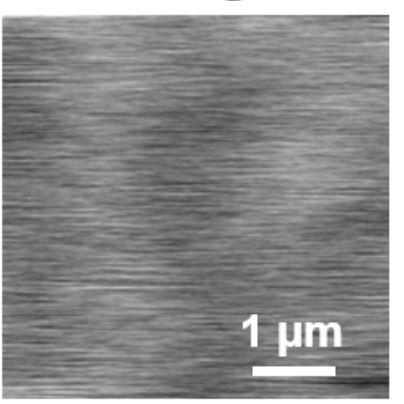

$500 \mathrm{~nm}$

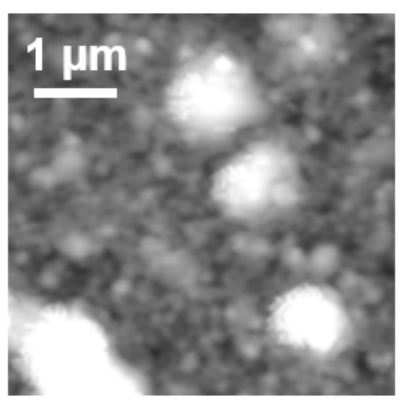

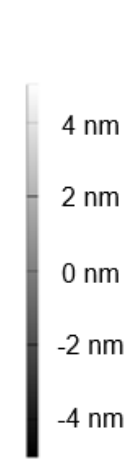

$-200 \mathrm{~nm}$

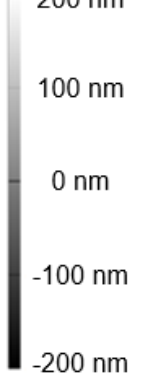

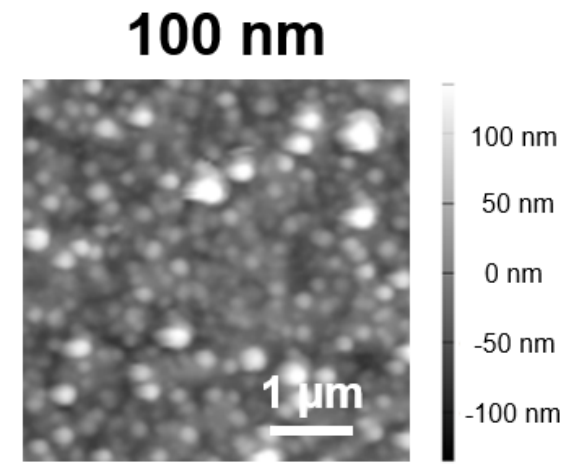

$2000 \mathrm{~nm}$

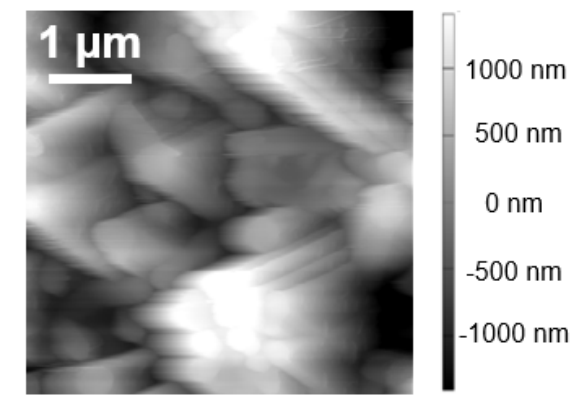

Figure S1. Heights of the nanofeatured substrates measured by atomic force microscopy (AFM).
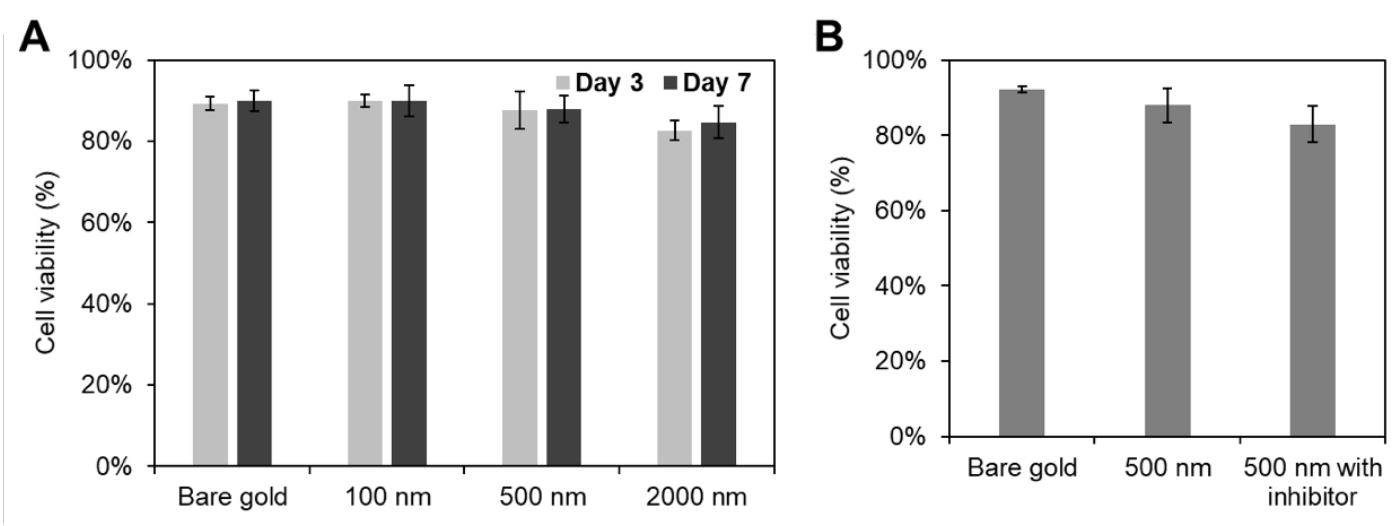

Figure S2. Quantification of cell viability (A) on-chip and (B) after CHIR treatment (n $=4)$. 

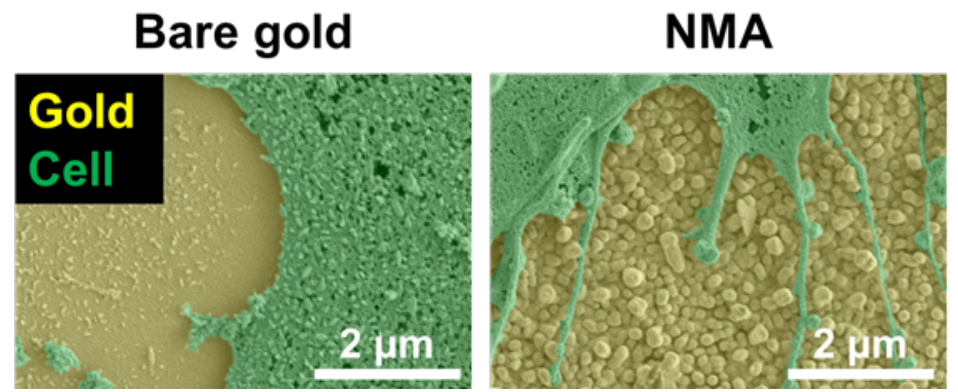

NMA, side view

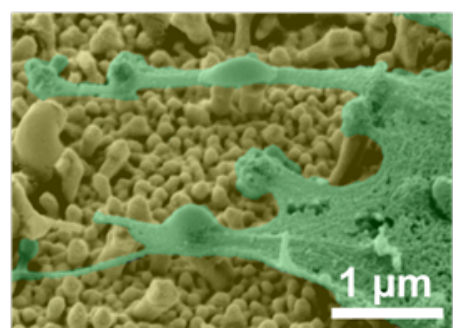

Figure S3. Representative scanning electron microscopy (SEM) images showing that the cells seeded on NMAs interact with the nanoscale features and form protrusions.
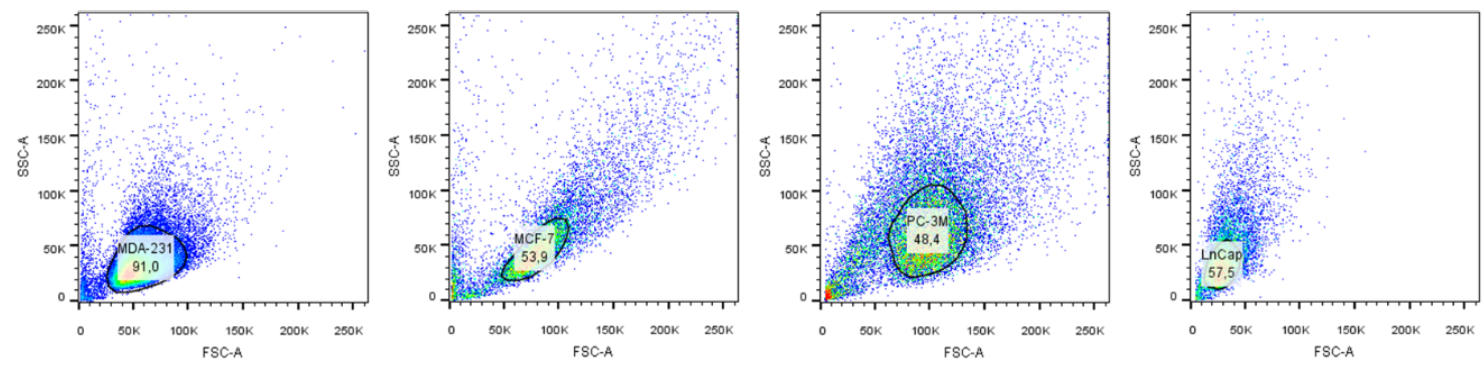

Figure S4. Representative images showing the gating strategy during flow cytometric analysis.

MCF-7

LnCap
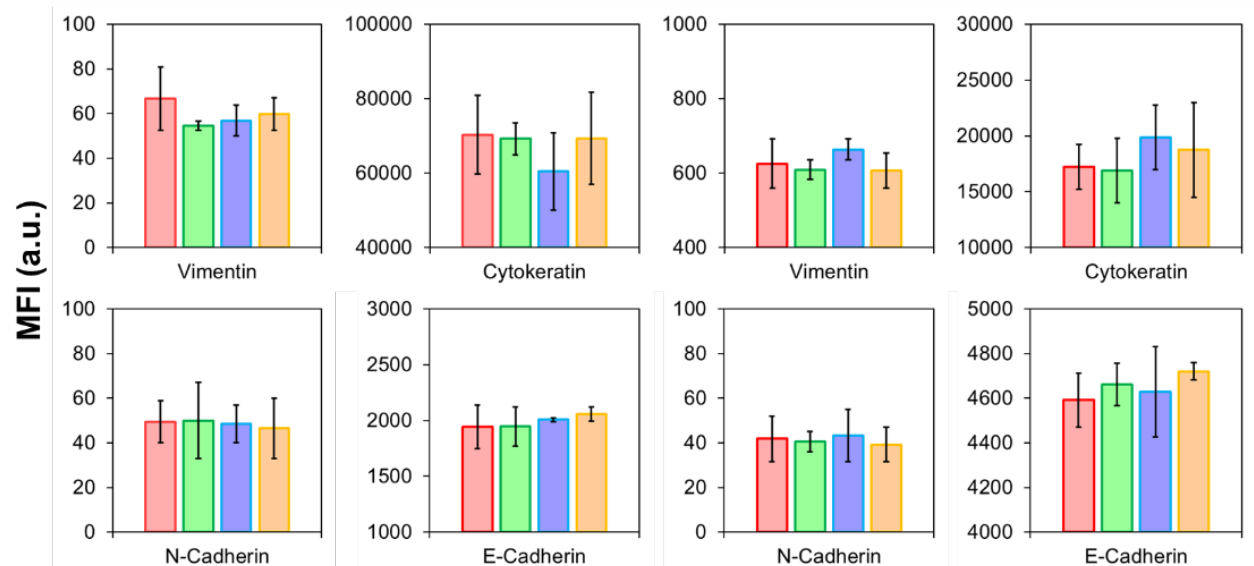

$\square$ Bare gold $\square 100 \mathrm{~nm} \quad \square 500 \mathrm{~nm} \square 2000 \mathrm{~nm}$

Figure S5. Quantification of median fluorescence intensity (MFI) of flow cytometric samples ( $n=2$, no statistical significance was found using ANOVA). 

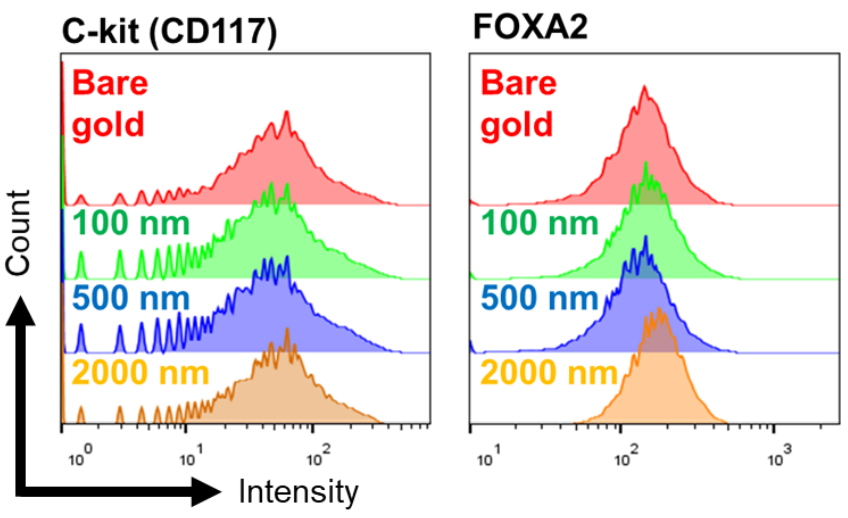

Figure S6. Flow cytometric analysis of on-chip cells: Stemness-related markers.
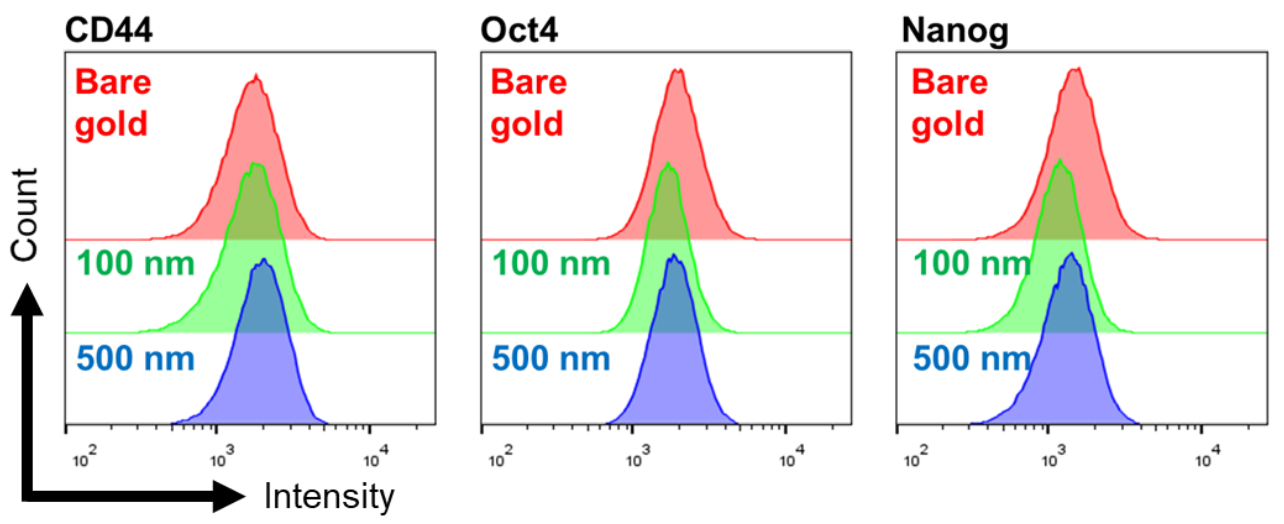

Figure S7. Flow cytometric analysis of MET cells cultured on flat surfaces: Stemness-related markers.

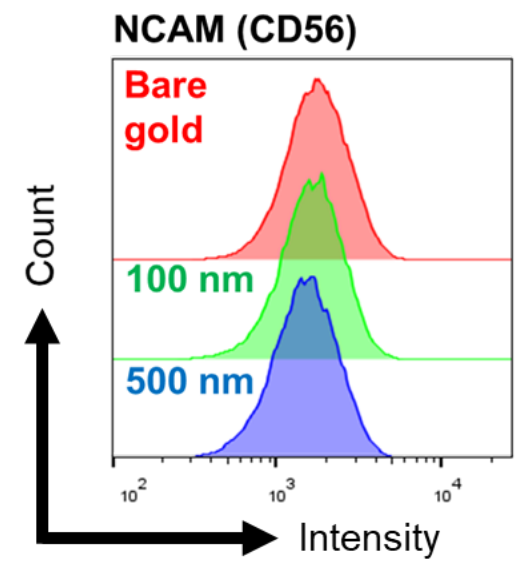

THY1 (CD90)

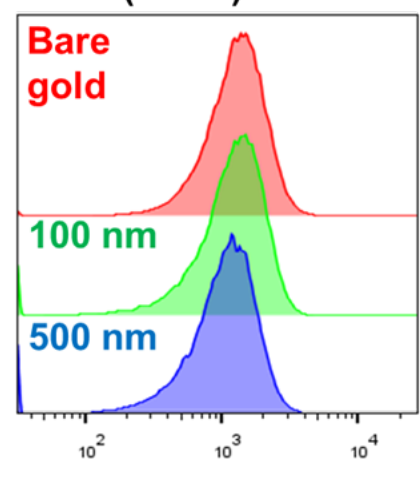

Figure S8. Flow cytometric analysis of MET cells cultured on flat surfaces: Neuroendocrine markers. 

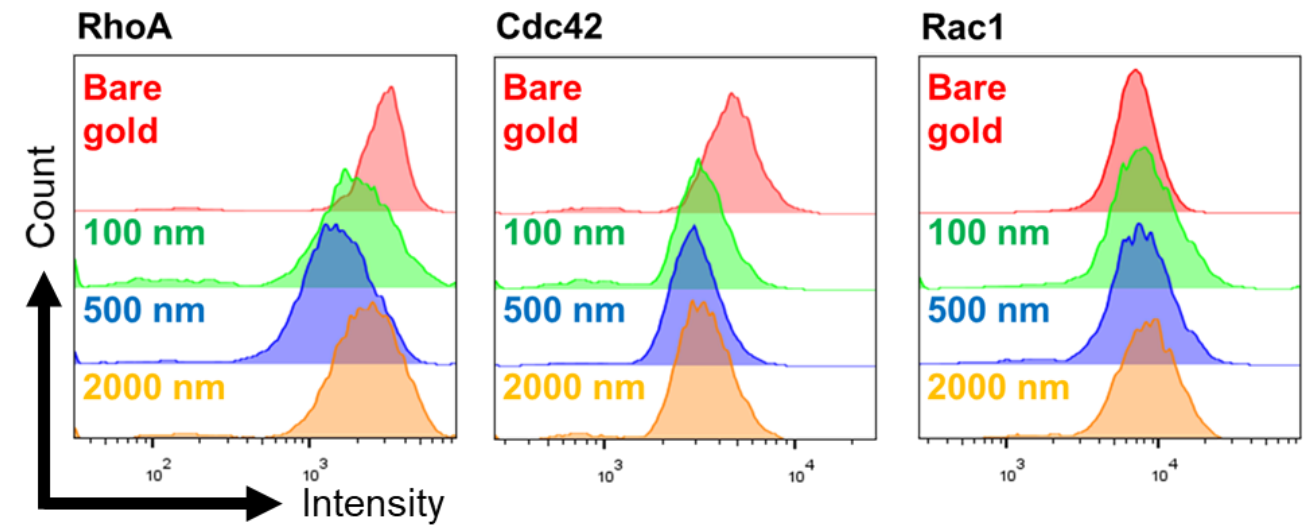

Figure S9. Flow cytometric analysis of on-chip cells: Key transcriptional factors for EMT/MET.

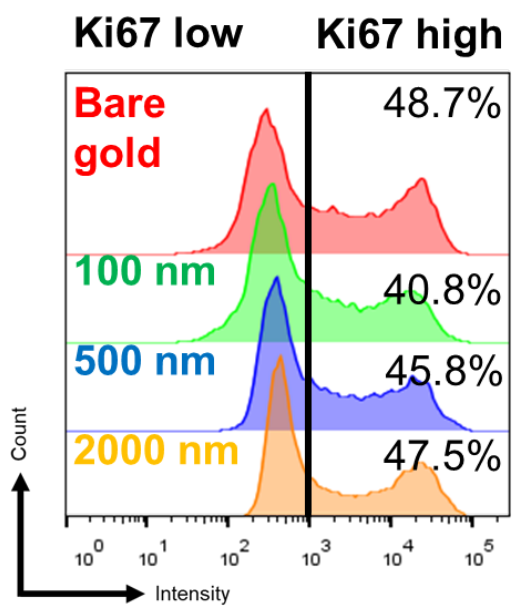

Figure S10. Flow cytometric analysis of cell proliferation on-chip. 

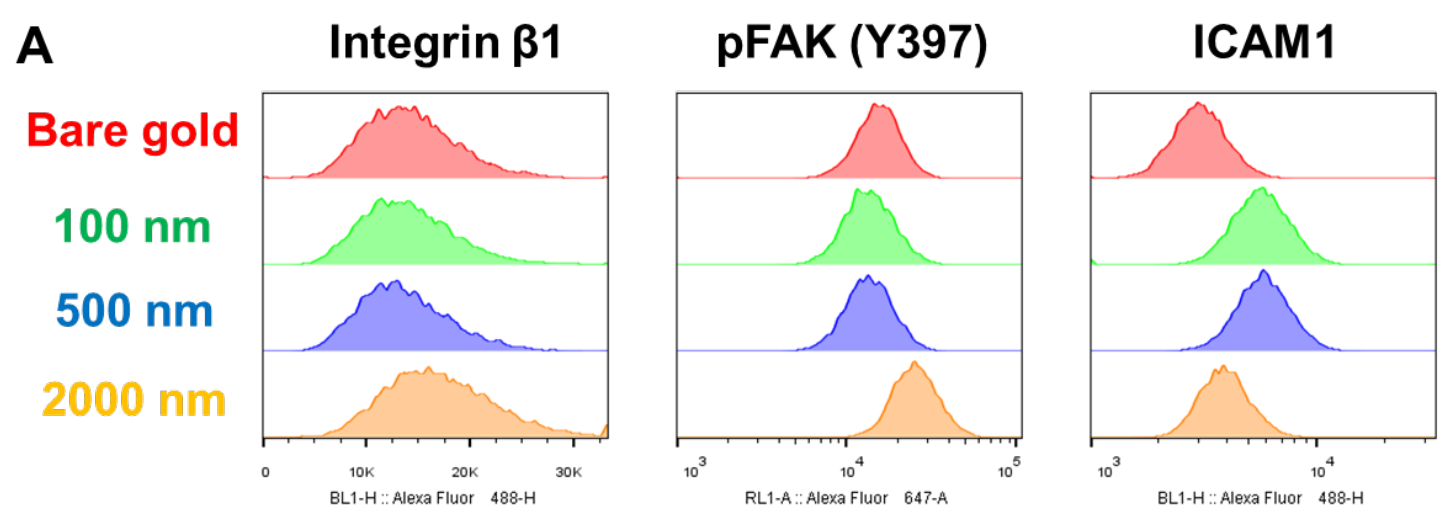

B

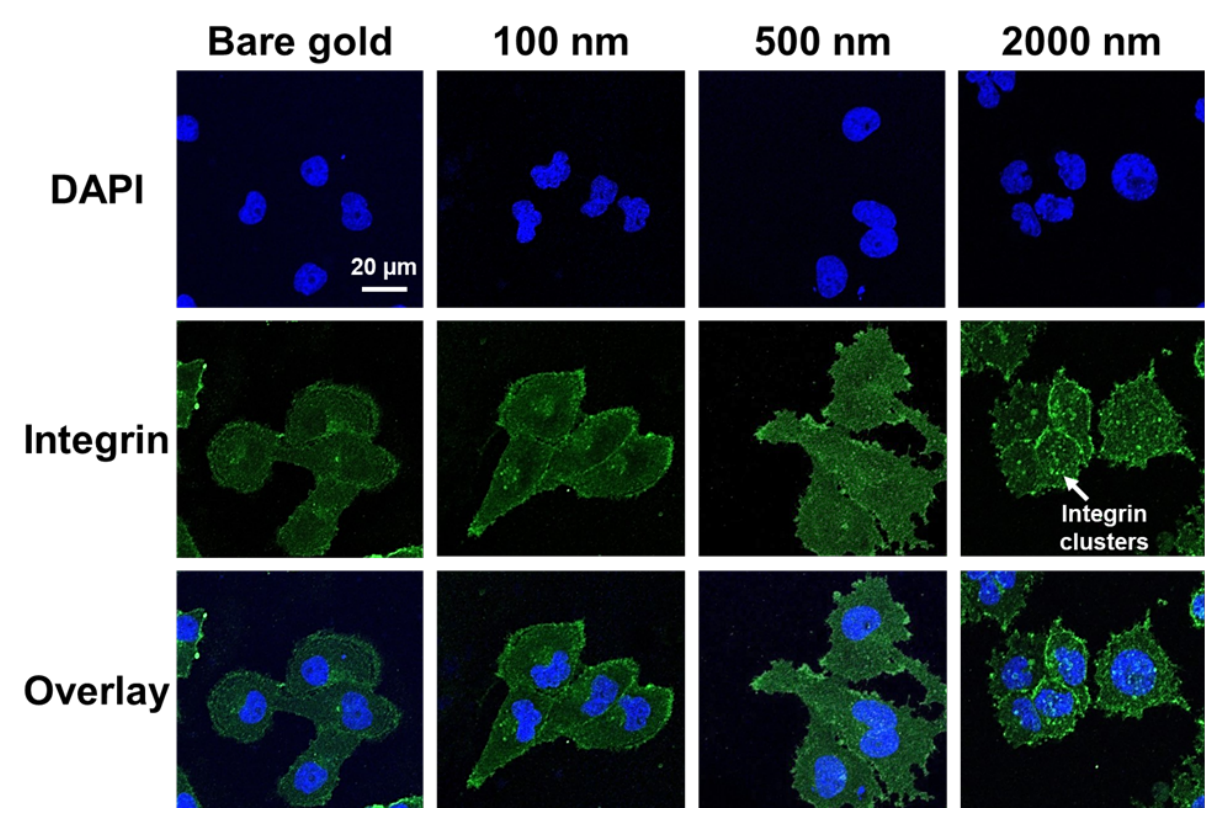

Figure S11. Microfeatures, not nanofeatures, promote the expression and clustering of integrin $\beta 1$ on-chip, which further activates the phosphorylation of focal adhesion kinase (pFAK) and downregulates ICAM1 expression. (A) Flow cytometric analysis of the expression of integrin $\beta 1$, pFAK and ICAM1 on different substrates. (B) Representative confocal images showing the clustering of integrin in response to different substrates. 


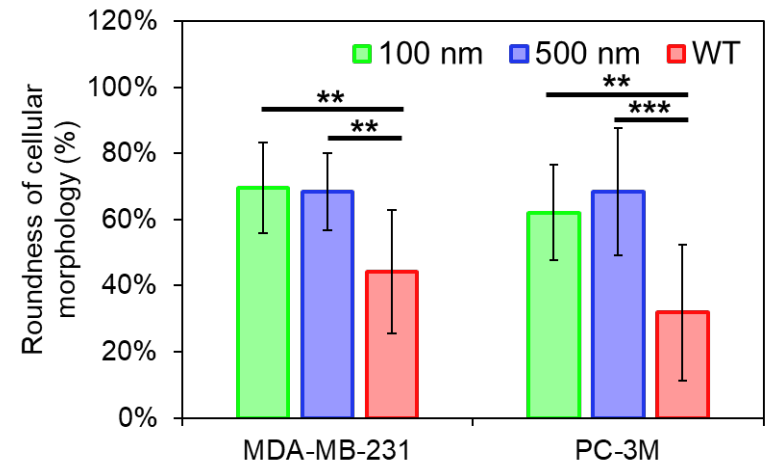

Figure S12. Quantitation of the roundness of WT and METed cells recultured on the flat substrates for 7 days $\left(n=10,{ }^{* *} p<0.01,{ }^{* * *} p<0.001\right)$.

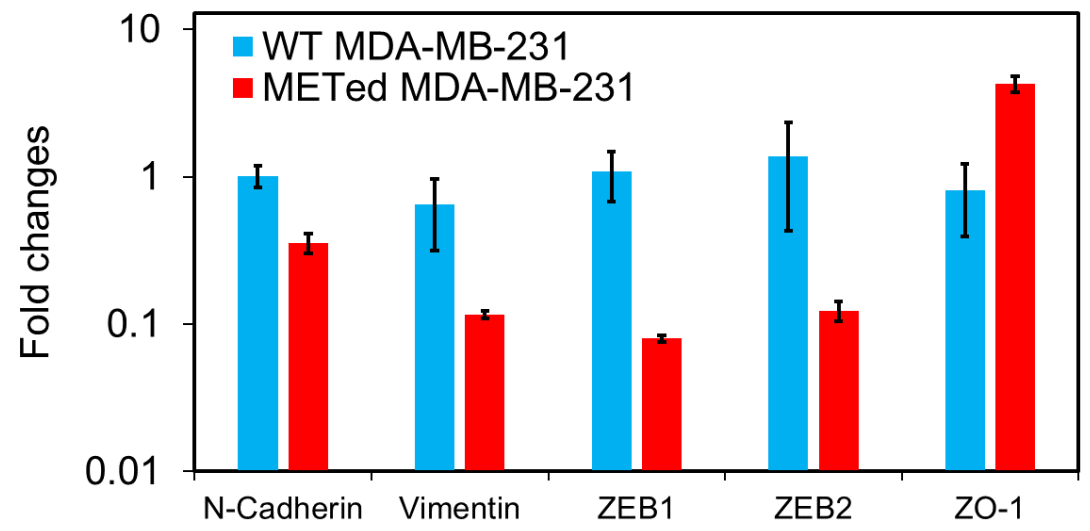

Figure S13. Analysis of EMT proteins at mRNA level $(n=3)$.
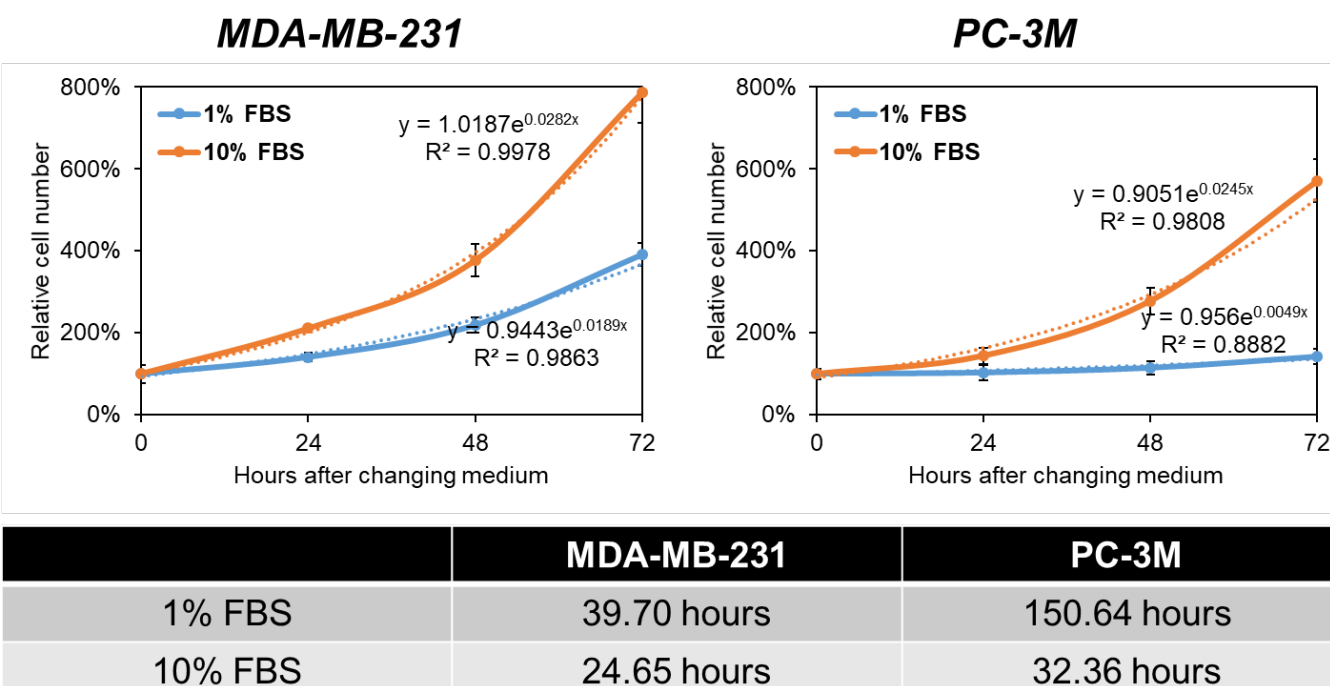

MDA-MB-231

PC-3M

$10 \%$ FBS

39.70 hours

150.64 hours

32.36 hours

Figure S14. Quantification of doubling time of MDA-MB-231 and PC-3M cells at normal and reduced serum condition $(n=4)$. 

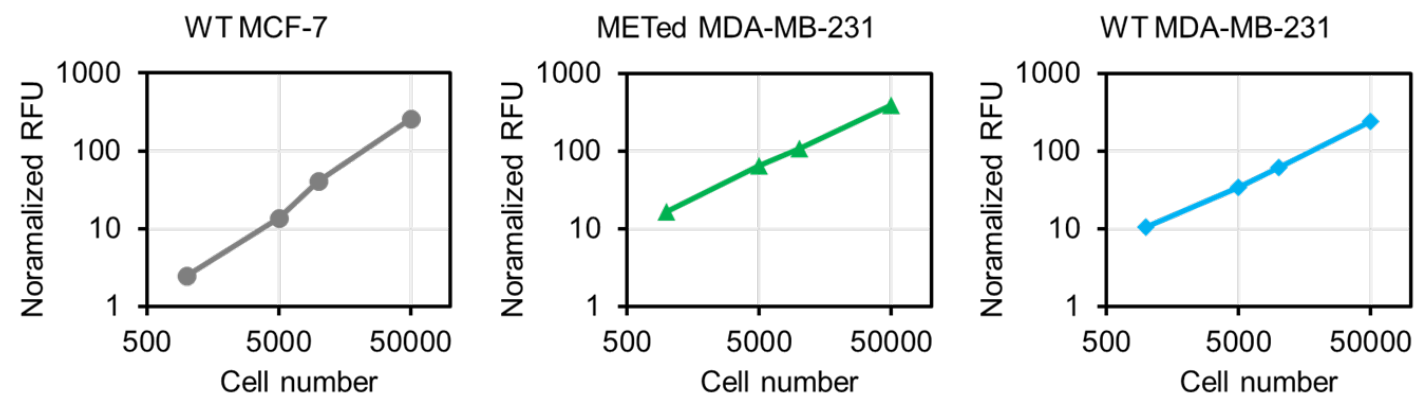

Figure S15. Normalization curve obtained experimentally to cover measured relatively fluorescence unit (RFU) to cell number. $R^{2}$ of each curve is higher than $\mathbf{0 . 9 9}$, indicating a high linearity of the Boyden chamber assay in the range of 1000 to 50,000 migrated cells.

Human Phospho-Kinase Array Coordinates

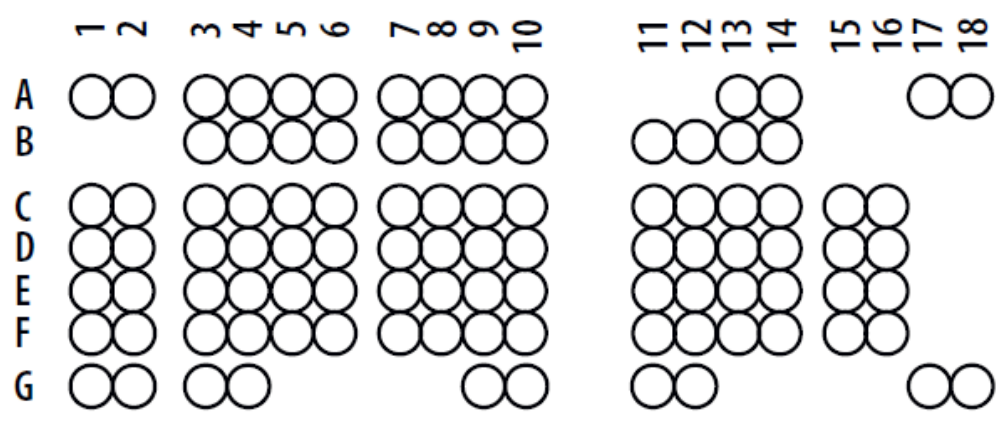

Figure S16. Allocation of spots on the proteome profiler used for phosphor-kinase analysis.

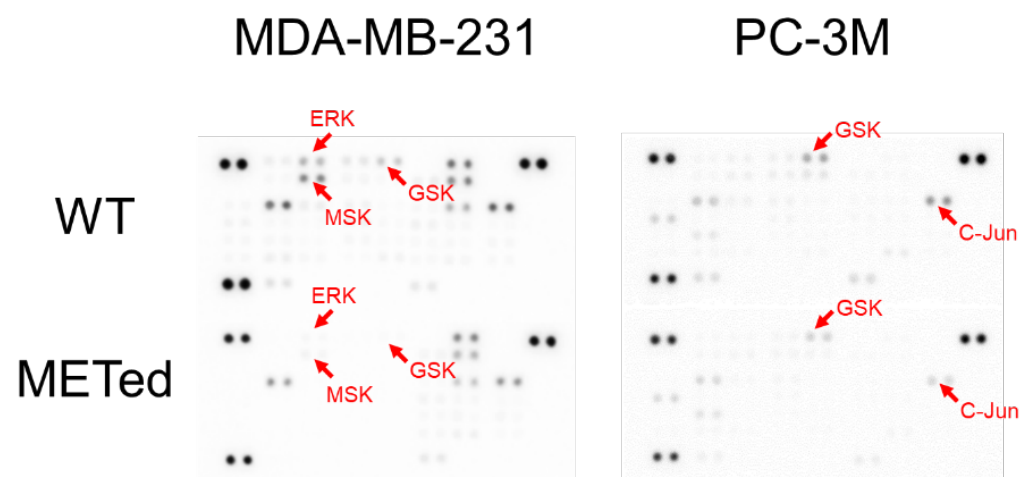

Figure S17. Representative images used for protein microarray analysis. Red arrows indicate the spots that were significantly changed. 